Case Report

\title{
MOYAMOYA disease-two case reports
}

\author{
Kundu GK ${ }^{1}$, Benzamin $M,{ }^{2}$ Kundu NK, ${ }^{3}$ Roy SK, ${ }^{4}$ Hossain MN, ${ }^{5}$ Rahman MM, ${ }^{6}$ Akter $S^{7}$
}

\section{Introduction}

Moyamoya disease is a chronic, progressive occlusion of the circle of Willis arteries that leads to the development of characteristic collateral vessels evidence by imaging, particularly cerebral angiography. ${ }^{1}$ The disorder initially involves the intracranial portion of the internal carotid arteries and progresses to involve the middle, anterior and posterior cerebral arteries. Collateral vascular network developed due to compensatory dilatation of lenticulostriate and thalamostriate arteries .Angiographic study of these collateral vascular network give rise to characteristics appearance such as a puff of cigarette smoke. The Japanese term "MOYAMOYA" means "some thing hazy like a puff of cigarette smok. ${ }^{2-4}$

Moyamoya disease was first described in Japan by Takeuchi and Shimizu in 1957. Though The disease is most common in Japan, many subsequent cases have been reported elsewhere Asia, Europe and North America. In 1988 the Japanese Ministry of Health and Welfare defined primary MMD as an idiopathic bilateral stenoses of arteries of the circle of Willis with collateral vascular networks, demonstrated on angiography.

The moyamoya syndrome (MMS) have same angiographic appearance as moya moya disease such as atherosclerosis, neurofibromatosis, postradiation therapy, sickle cell disease, systemic lupus erythromatosus and down syndrome (DS). ${ }^{1}$

1. *Dr Gopen Kumar Kundu, Associate Professor, Institute of Paediatric Neuro disorder and Autism (IPNA),

Bangabandhu Sheikh Mujib Medical University Dhaka, Email: gopen.kundu@gmail.com

2. Dr Md Benzamin, Resident, Department of Paediatric Gastroenterology \& Nutrition, Bangabandhu Sheikh Mujib Medical University, Dhaka

3. Dr Nripen Kumar Kundu, Registrar, Department of Orthopedic Surgery, Popular Medical College, Dhaka

4. Dr Subrota Kumar Roy, Assistant Professor, Department of Paediatrics, Dhaka Medical College, Dhaka

5. Dr Md Nasir Hossain, Assistant Professor, Dept of Paediatrics, Dhaka Medical College, Dhaka

6. Professor Dr Md Mizanur Rahman, Professor, Institute of Paediatric Neuro disorder and Autism, Bangabandhu Sheikh Mujib Medical University, Dhaka

7. Professor Dr Shaheen Akter, Professor \& Director, Institute of Paediatric Neuro disorder and Autism Bangabandhu Sheikh Mujib Medical University, Dhaka

*For correspondence

\section{Case report-1}

A three years old immunized girl of non consanguineous parents came to paediatric neurology of Bangabandhu Sheikh Mujib Medical University, Dhaka with left sided hemiperesis for 1 year. Mother stated that she developed seizure followed by left sided hemiplegia to extent that she unable to stand or walk. After this episode she gradually improved in standing $\&$ walking but weakness persist that made her walking difficult. Her perinatal and family history was normal. Milestone of developement was age appropriate prior to this illness. There was no history of fever, unconsiousness, vomiting, or aphasia along with this illness and no history of radiation exposure or head trauma mentioned before this. On examination she had no facial dysmorphism, vitals were within normal limit, skin survey-normal, no chest murmur. Neurological findings revealed that she had hemiplegic gait, normal tone, power $4 / 5$ on left side (both upper \& lower limb) along with hyperreflexia,exaggrarated ankle clonus \& babinski sign positive. Cranial nerve \& sensory function, cerebeller function were intact.

Among the investigation, complete blood count, chest radiograph, ECG \& EEG were normal. MRI of brain flare image showed bilateral chronic cerebral ischemic changes, more remarkable on right side. (Figure-1) MRA showed

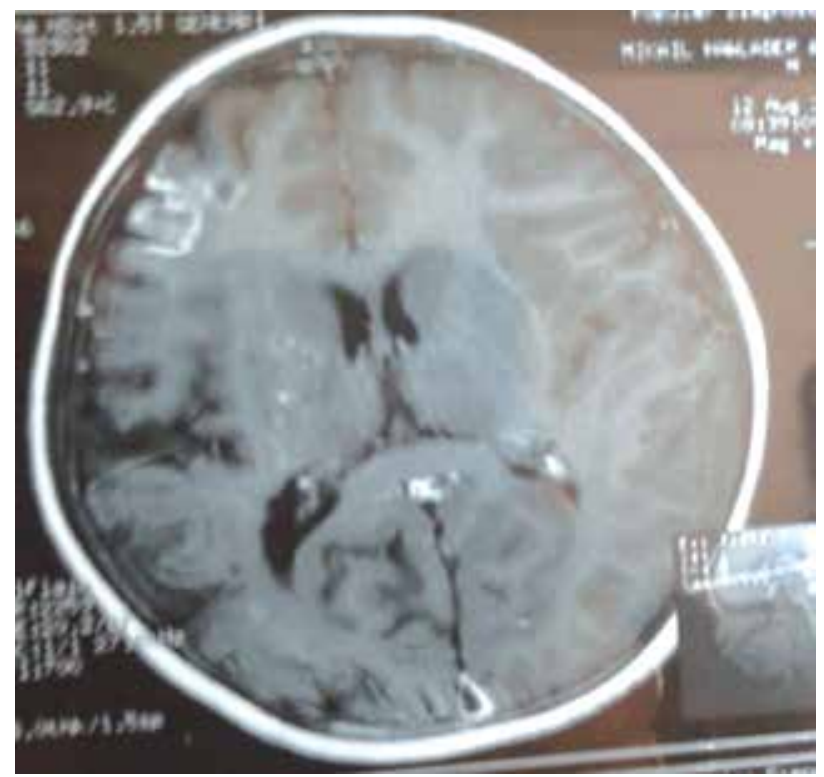

Figure-1: MRI of brain (flare image) shows chronic cerebral ischemic changes, more remarkable on right side. 
gross narrowing of anterior cerebral, middle cerebral \& posterior cerebral artery with extensive collaterals that appears as puff of smoke appearence suggestive of MOYAMOYA disease. (Figure-2 \& 3) This patient was treated medically by nifidipine as vasodilator and ecospirin and patient become gradually ambulant. Then we planned to discharge the patient after neurosurgical consultation.

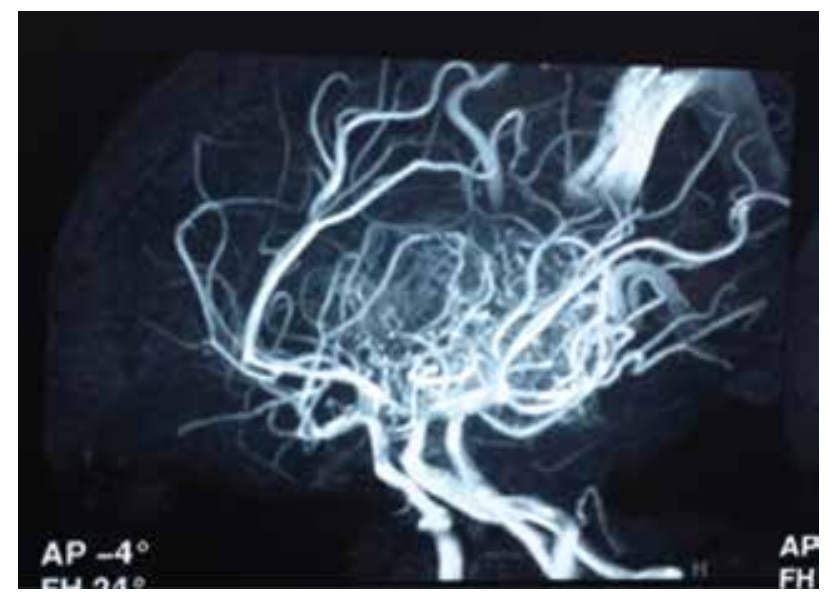

Figure-2: MR angiogram (right oblique veiw) shows characterstic Moyamoya vessels with typical "puff of smoke" appearance

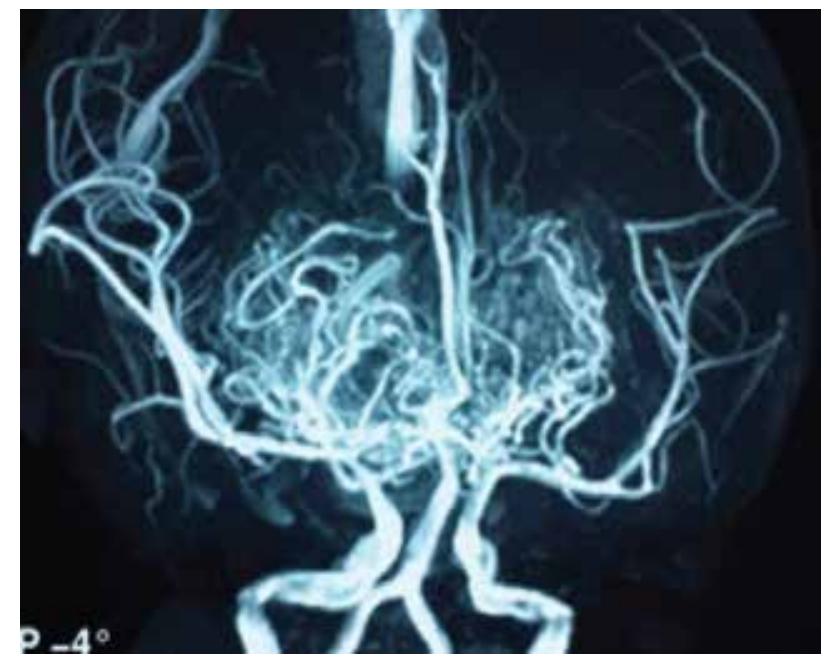

Figure-3: MR angiogram (AP veiw) shows stenosis of bilateral internal carotid arteries

\section{Case report- 2}

A 5 years old immunized boy of consanguineous mating parents came from rural area of Bangladesh presented with left sided hemiperesis for last 5 days, was not associated with fever, unconsiousness or aphasia. He had history of repeated attack of frontal, throbbing headache, associated with vomiting. He had an episode of chicken pox 1 month back. His perinatal history and milestone of development was normal. Examination findings reveals that he had no facial dysmorphism, vitals were within normal limit ,multiple post vericella healed skin lesion found in whole body, no signs of meningeal irritation present there. Neurological findings -hypotonia, grade 3/5 power on left side (both upper \& lower limb) with areflexia, bilateral planter flexor, hemiplegic gait were present. Cranial nerve \& sensory function, cerebeller functions were normal.

Among the biochemical reports, complete blood count, blood glucose, Lipid profile, serum lactate, Blood ammonia, renal function tests, liver function tests, CXR \& ECG were within normal limit. CT scan of brain shows-small infract in right parital region. MRI of brain T2 and flair image multiple flow voids in the area of circle of willis and bilateral basal ganglia.(Figure-4\&5) MR angiogram showed bussy change in the both parietal region. (Figure-6\&7) He was diagnosed as moyamoya disease. The patient was treated by methylprednisolone for 3 days followed by oral steroid along with aspirin. Patient was gradually improved $\&$ discharged with advice for regular follow up.

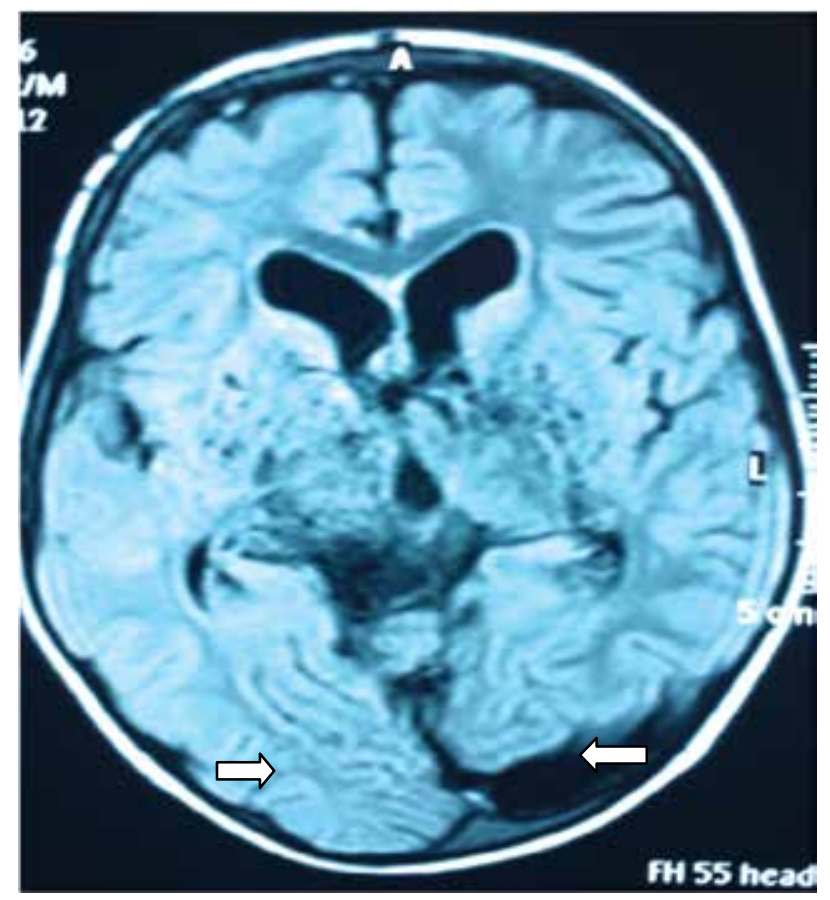

Figure-4: MRI of brain flair image showed multiple flow voids in basal ganglia region(arrow) 


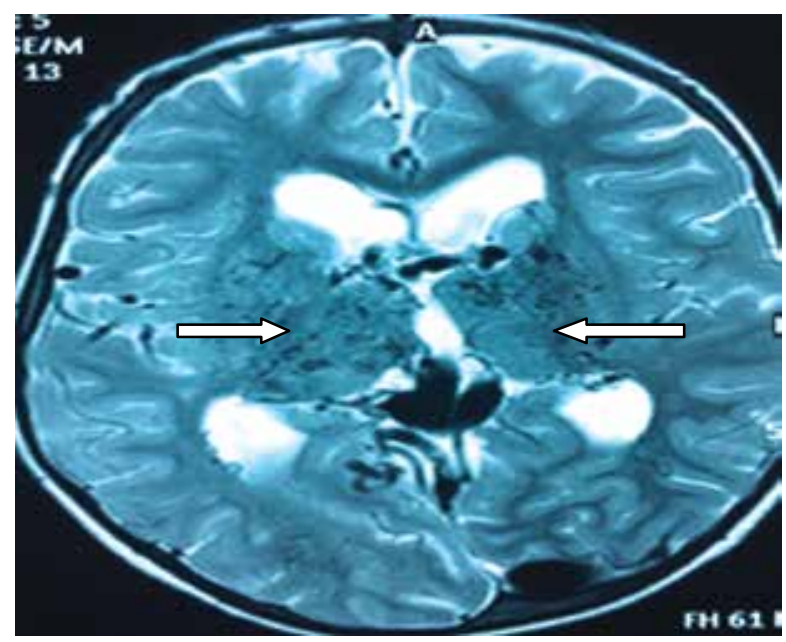

Figure-5: MRI of brain T2 image showed multiple flow voids in the bilateral basal ganglia(arrow)

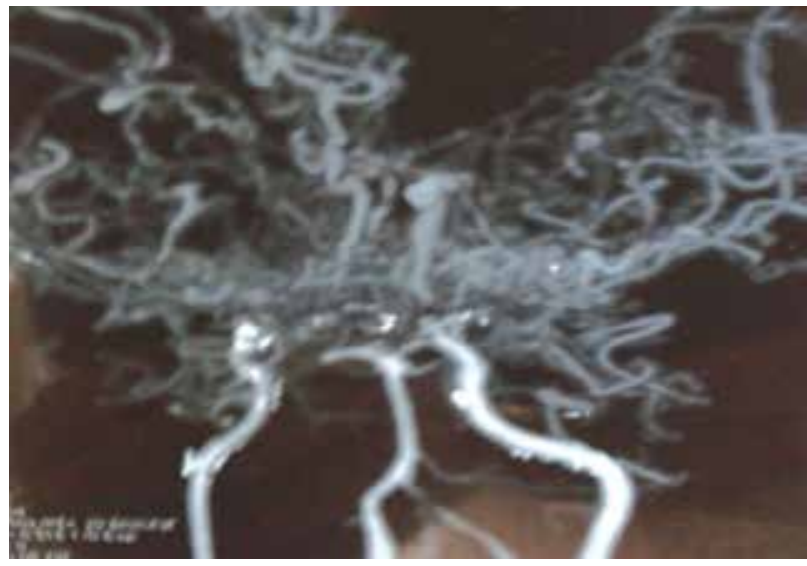

Figure-6: MR angiogram (A-P veiw) shows bilateral stenosis of internal carotid arteries with typical "puff of smoke" appearance

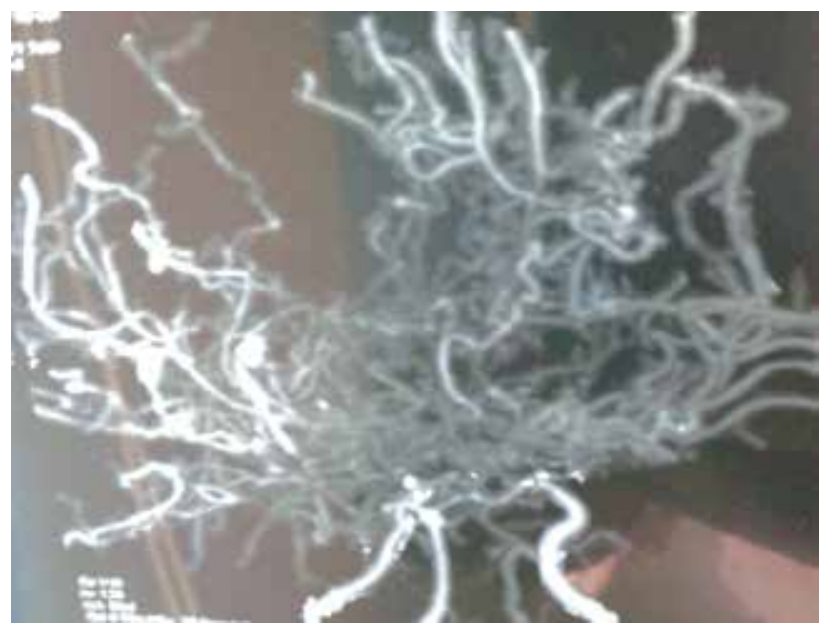

Figure-7: MR angiogram (right oblique veiw) shows characterstic Moyamoya vessels with typical "puff of smoke" appearance

\section{Discussion}

MoyaMoya disease is a rare idiopathic, slowly progressive vasculopathy characterized by irreversible stenosis of the arteries of Circle of Willis leading to cerebral hypoperfusion. The occlusive process stimulates the development of an extensive network of enlarged basal transcortical and transdural collateral vessels.

MoyaMoya disease was first reported in Japan in 1957 and has since been reported worldwide. ${ }^{4-7}$ The disease has a particularly high incidence in Eastern Asia, especially in Japan. ${ }^{6}$ The overall prevalence rate of moyamoya disease in Japan in 1995 was 3.16 per 100,000 with an incidence rate of 0.35 per $100,000.4,5$ The male and female ratio is $1.8: 1$ and a family history of moyamoya is noted in $10 \%$ of cases. ${ }^{5,6}$

The exact etiologic process of moyamoya disease is unknown, though myriad bacterial, environmental, genetic, and viral causes have been theorized. ${ }^{3}$

Family occurrence suggests that a genetic predisposition is present. ${ }^{4,5}$ Elevated levels of cellular retinoic acid-binding protein-I were found in the cerebrospinal fluid of patients with moyamoya disease. ${ }^{8}$ High levels of hepatocyte growth factor, a known inducer of angiogenesis, have been found in the carotid fork and cerebrospinal fluid of patients with moyamoya disease. ${ }^{9}$

Pathological changes in moyamoya patients include intimal thickening with fibrous tissue, abnormalities of internal lamina elastica, variable lipid deposition and virtual absence of inflammatory reaction in the blood vessel. ${ }^{10,11}$

Clinically, the presentation of patients with moyamoya disease may include seizures, transient ischemic attacks, ischemic strokes, and hemorrhagic strokes. ${ }^{12,13,14}$ Visual deficits, speech disturbance, migrane like headache, intellectual deterioration, cranial nerve palsies, and disturbance of gait can also be evident. ${ }^{15,16}$ Our both patient presented with ishchemic stroke and hemiplegia

Conventional angiogram or Digital subtraction angiogram ( DSA) is the investigation of choice. MRA has a reliable diagonostic modality for MMD. ${ }^{7}$ CT and MRI are usefull for detecting region of cerebral infraction. MRA defines the extent of disease and to some extent, the adequency of collateral flow. It typically reveals the narrowing and occlusion of proximal cerebral vessels and extensive collateral flow through the perforating vessels demonstrating the classic 'puff of smoke' appearance. 
T2W1 of MRI of our patient showedmultiple slow voids at and around the base of the brain. Angiogram of both patient revealed stenosis of bilateral carotid systems as well as the typical 'puff of smoke' appearance of collateral vessels

Treatment in the acute phase is symptomatic with the goal of maintaining cerebro-vascular perfusion and function. Anticoagulant and antiplatelet agents have shown no remarkable benefit. ${ }^{17}$ The same lack of obvious efficacy has been described for corticosteroids in moyamoya disease. ${ }^{18}$ McLean et al, elucidated the use of verapamil hydrochloride to curtail the ischemic symptoms associated with moyamoyadisease ${ }^{19}$, Surgical treatment modalities have been used to manage the hemorrhagic and ischemic consequences of moyamoya disease. ${ }^{4,10,20-22}$ Direct revascularization techniques, which are typically used in adults, include the superficial temporal artery to middle cerebral artery bypass or the middle meningeal artery to middle cerebral artery bypass are commonly applied in children. ${ }^{21,22}$

Although Moyamoya is predominat in Japanese population but should not overlooked in other population. The patient who will fulfill the clinical characteristics, MR angiogram should be done to diagnose Moyamoya disease.

\section{References}

1. Fukushima Y, Kondo Y, Kuroki Y, Miyake S, Iwamoto H. Are Down syndrome patients predisposed to moyamoya disease? Eur J Pediatr. 1986; 144 : 516-7.

2. Takebayashi S., Matsuo K., Kaneko M. Ultrastructural studies of cerebral arteries and collateral vessels in moyamoya disease. Stroke. 1984; 15 : 728-32

3. Ishimori ML, Cohen SN, Hallegua DS, Moser FG, Weisman $\mathrm{MH}$. Ischemic stroke in a postpartum patient: understanding the epidemiology, pathogenesis, and outcome of moyamoya disease. Semin Arthritis Rheum. 2006;35:250-9

4. Uchino K, Johnston SC, Becker KJ, Tirschwell DL. Moyamoya disease in Washington State and California. Neurology. 2005;65:956-8.

5. Yamauchi T, Houkin K, Tada M, Abe H. Familial occurrence of moyamoya disease. Clin Neurol Neurosurg. 1997;99(2):S162-S167.

6. Ikeda H, Sasaki T, Yoshimoto T, Fukui M, Arinami T. Mapping of a familial moyamoya disease gene to chromosome . Am J Hum Genet. 1999;64:533-7.
7. Sakurai K, Horiuchi Y, Ikeda H, Ikezaki K, Yoshimoto T, Fukui $\mathrm{M}$ et al. A novel susceptibility locus for moyamoya disease on chromosome 8q23. J Hum Genet. 2004;49:278-81.

8. Kim SK, Yoo JI, Cho BK, Hong SJ, Kim YK, Moon JA et al. Elevation of CRABP-I in the cerebrospinal fluid of patients with moyamoya disease. Stroke. 2003;34:2835-41.

9. Nanba R, Kuroda S, Ishikawa T, Houkin K, Iwasaki Y. Increased expression of hepatocyte growth factor in cerebrospinal fluid and intracranial artery in moyamoya disease. Stroke. 2004;35:2837-2842.

10. Yilmaz EY, Pritz MB, Bruno A, Lopez-Yunez A, Biller J. Moyamoya: Indiana University Medical Center experience. Arch Neurol. 2001;58:1274-77.

11. Inoue TK, Ikezaki K, Sasazuki T, Matsushima T, Fukui M. Analysis of classII genes of human leukocyte antigen in patients with moyamoya disease. Clin Neurol Neurosurg. 1997;99(suppl 2):S234-S237.

12. Bertora P, Lovati C, Gambaro P, Vicenzi A, Rosa S, Osio $\mathrm{M}$, et al. Moyamoya disease in a member of the Roma gypsy community. Eur Neurol. 2008:59: 274-5.

13. Wakai K, Tamakoshi A, Ikezaki, Fukui M, Kawamura $\mathrm{T}$, Aoki R et al. Epidemiological features of moyamoya disease in Japan: findings from a nationwide survey. Clin Neurol Neurosurg. 1997;99(2):S1-S5.

14. Fukui M. Research Committee on Spontaneous Occlusion of the Circle of Willis (Moyamoya disease) of the Ministry of Health and Welfare, Japan. Guidelines for the diagnosis and treatment of spontaneous occlusion of the circle of Willis. Clin Neurol Neurosurg. 1997; 99:S238-S240

15. Kitamura K, Fukui M, Oka K. Moyamoya disease. In: Handbook of Clinical Neurology. Vol 2. Amsterdam, The Netherlands: Elsevier; 1989:293-306

16. Hoare AM, Keogh AJ. Cerebrovascular moyamoya disease. Br Med J.1974;1:430-432

17. Takanashi J, Sugita K, Honda A, Numi H. Moyamoya syndrome in a patient with Down syndrome presenting with chorea. Pediat. Neurol. 1993; 9 : 396-98.

18. Spittler JF, Smektala K. Pharmacotherapy in moyamoya disease. HokkaidoIgaku Zasshi. 1990; 65: 235-40. 
19. McLean MJ, Gebarski SS, vander Spek AF, Goldstein GW. Response of moyamoya disease to verapamil. Lancet. 1985;1:162-3.

20. Chiu D, Shedden P, Bratina P, Grotta JC. Clinical features of moyamoya disease in the United States. Stroke. 1998;29:1347-51.

21. Kuroda S, Ishikawa T, Houkin K, Nanba R, Hokari M, Iwasaki Y. Incidence and clinical features of disease progression in adult moyamoya disease. Stroke. 2005; 36: 2148-53.

22. Oya S, Tsutsumi K, Ueki K. Adult-onset moyamoya disease with repetitive ischemic attacks successfully treated by superficial temporal-middle cerebral artery bypass - case report. Neurol Med Chir. 2003; 43: 138-141. 